\title{
Ширина линии излучения и $\alpha$-фактор одномодовых вертикально-излучающих лазеров спектрального диапазона 850 нм на основе квантовых ям InGaAs/AIGaAs *
}

\author{
(C) С.А. Блохин ${ }^{1}$, М.А. Бобров ${ }^{1}$, А.А. Блохин ${ }^{1}$, А.Г. Кузьменков ${ }^{2,1}$, А.П. Васильев ${ }^{2,1}$, \\ Ю.М. Задиранов ${ }^{1}$, Е.А. Европейцев ${ }^{1}$, А.В. Сахаров ${ }^{1}$, Н.Н. Леденцов ${ }^{3}$, \\ Л.Я. Карачинский ${ }^{4,1}$, А.М. Оспенников ${ }^{5}$, Н.А. Малеев ${ }^{1}$, В.М. Устинов ${ }^{2,1,6}$ \\ ${ }^{1}$ Физико-технический институт им. А.Ф. Иоффре Российской академии наук, \\ 194021 Санкт-Петербург, Россия \\ ${ }^{2}$ Научно-технологический центр микроэлектроники и субмикронных гетероструктур \\ Российской академии наук, \\ 194021 Санкт-Петербург, Россия \\ ${ }^{3}$ VI Systems GmbH, \\ Berlin, Germany, D-10623 \\ ${ }^{4} \mathrm{OOO} \mathrm{„Коннектор} \mathrm{Оптикс“,}$ \\ 194292 Санкт-Петербург, Россия \\ ${ }^{5} \mathrm{AO}$ „РИРВ“, \\ 192012 Санкт-Петербург, Россия \\ ${ }^{6}$ Санкт-Петербургский политехнический университет Петра Великого, \\ 195251 Санкт-Петербург, Россия \\ E-mail: blokh@mail.ioffe.ru
}

(Получена 25 мая 2017 г. Принята к печати 30 мая 2017 г.)

\begin{abstract}
Проведены исследования ширины линии излучения одномодовых вертикально-излучающих лазеров спектрального диапазона 850 нм на основе квантовых ям InGaAs/AlGaAs. Ширина линии излучения лазера с характерным размером оксидной токовой апертуры 2 мкм достигает своего минимума $\sim 110$ МГц при выходной мощности 0.8 мВт. При дальнейшем повышении выходной оптической мощности наблюдается аномальное уширение линии излучения, что, по-видимому, обусловлено ростом $\alpha$-фактора вследствие падения дифференциального усиления активной области в условиях повышенной концентрации носителей и высоких внутренних оптических потерь в микрорезонаторе. Проведена оценка величины $\alpha$-фактора двумя независимыми методами.
\end{abstract}

DOI: 10.21883/FTP.2018.01.45326.8657

\section{1. Введение}

Полупроводниковые вертикально-излучающие лазеры (ВИЛ, vertical-cavity surface-emitting lasers - VCSELs) спектрального диапазона 850 нм широко применяются как для оптической передачи данных, так и в различных типах сенсоров и датчиков [1]. Большое внимание уделяется вопросу создания сверхскоростных энергоэффективных оптических межсоединений, когда необходимо повысить быстродействие лазеров в режиме прямой токовой модуляции, снизить их энергопотребление и одновременно уменьшить ширину спектра лазерного излучения для снижения дисперсии сигнала при передаче данных по оптическому волокну [2,3]. В последние годы растет интерес к микроминиатюрным источникам лазерного излучения на основе ВИЛ ближнего инфракрасного (ИК) диапазона для применения в квантовых магнитометрах [4,5], лазерных интерферометрах [6] и квантовых стандартах частоты (так называемые атомные часы) [7,8], где принципиально требуется обеспечить одномодовый режим лазерной генерации ВИЛ с фик-

\footnotetext{
* Полный текст этой статьи будет опубликован в журнале „Физика и техника полупроводников“, том 52 вып. 1.
}

сированным направлением поляризации и малой шириной линии излучения. Классический способ получения одномодового режима лазерной генерации ВИЛ уменьшение размеров токовой апертуры, используемой для ограничения области инжекции носителей. В ВИЛ с токовой апертурой, сформированной методом ионной имплантации, ввиду малого скачка эффективного коэффициента преломления отсутствует стабильное поперечное оптическое ограничение и модовый состав лазерного излучения определяется тепловыми эффектами и/или пространственным выжиганием дырок, а безызлучательная рекомбинация на радиационных дефектах ведет к резкому росту порогового тока с уменьшением размеров апертуры. Указанные факторы не позволяют реализовывать таким способом эффективные одномодовые быстродействующие лазеры. Применение токовой апертуры, полученной методом селективного окисления слоев $\mathrm{AlGaAs}$ с высоким содержанием $\mathrm{Al}$, позволяет обеспечить эффективное токовое и оптическое ограничение без существенного увеличения внутренних оптических потерь [1]. В коммерчески доступных одномодовых ВИЛ спектрального диапазона 850 нм на основе квантовых ям $\mathrm{GaAs} / \mathrm{AlGaAs}$, как правило, используется классическая 
геометрия микрорезонатора с инжекцией носителей заряда через легированные полупроводниковые распределенные брэгговские отражатели (РБО) и одной оксидной токовой апертурой. В этом случае типичная ширина линии лазерной генерации составляет $\sim 100$ МГц (при выходной мощности $\sim 0.5 \mathrm{MB}$ ). Увеличение эффективной длины микрорезонатора и уменьшение потерь на вывод излучения позволило снизить ширину линии генерации ВИЛ спектрального диапазона 850 нм до уровня $23 \mathrm{MГц} \mathrm{[9].} \mathrm{Однако} \mathrm{такой} \mathrm{подход} \mathrm{сопряжен} \mathrm{с} \mathrm{падением}$ быстродействия лазера (вследствие увеличения коэффициента затухания релаксационных колебаний), а также с уменьшением достижимого уровня выходной оптической мощности. С учетом современных тенденций в развитии конструкций ВИЛ ближнего ИК диапазона, связанных с увеличением дифференциального усиления активной области (напряженные активные области InAlGaAs) и снижением паразитной емкости (введение дополнительных оксидных слоев), актуальной задачей является анализ ширины линии генерации быстродействующих одномодовых ВИЛ на основе напряженных InAlGaAs квантово-размерных гетероструктур.

В настоящей работе представлены результаты экспериментальных исследований одномодовых поляризационно-стабильных ВИЛ спектрального диапазона 850 нм на основе квантовых ям (КЯ) InGaAs/AlGaAs в классической геометрии микрорезонатора с оксидной токовой апертурой, для которых обнаружено аномальное поведение ширины линии генерации при высоких уровнях выходной оптической мощности, предложено объяснение данного эффекта и проведены оценки величины $\alpha$-фактора.

\section{2. Детали эксперимента}

Эпитаксиальная структура исследуемых ВИЛ спектрального диапазона 850 нм представляет собой классический вертикальный микрорезонатор с выводом излучения вверх и состоит из скрытого контактного слоя $n^{+}$-GaAs, нижнего легированного распределенного брэгговского отражателя (РБО), содержащего 35 пар четвертьволновых слоев $\mathrm{Al}_{0.15} \mathrm{Ga}_{0.85} \mathrm{As} / \mathrm{Al}_{0.9} \mathrm{Ga}_{0.1} \mathrm{As}$ n-типа проводимости, микрорезонатора $1 \lambda$-AlGaAs с напряженной активной областью InGaAlAs, верхнего РБО, содержащего 22 пары четвертьволновых слоев $\mathrm{Al}_{0.15} \mathrm{Ga}_{0.85} \mathrm{As} / \mathrm{Al}_{0.9} \mathrm{Ga}_{0.1}$ As $p$-типа и поверхностного контактного слоя GaAs $p$-типа.

Для обеспечения быстрого захвата носителей и малого коэффициента компрессии усиления использована конструкция оптического мирокрорезонатора с раздельным ограничением носителей и градиентным профилем показателя преломления. Для повышения дифференциального усиления и подавления термического выброса носителей в качестве активной области использованы 5 слоев КЯ $\operatorname{In}_{0.08} \mathrm{Ga}_{0.92} \mathrm{As}$ толщиной 4 нм, окруженных барьерными слоями AlGaAs [10]. Число и толщина на- пряженных КЯ InGaAs, а также толщина барьера AlGaAs выбраны таким образом, чтобы обеспечить максимальный фактор оптического ограничения при сохранении положения пика усиления вблизи 850 нм без заметного ухудшения структурного и оптического качества КЯ. Для решения проблемы транспорта носителей через РБО использованы градиентные интерфейсы с модулированным профилем легирования на гетерограницах отдельных четвертьволновых слоев. Два апертурных слоя AlGaAs p-типа проводимости со ступенчатым профилем состава (содержания по Al) расположены в верхнем РБО в непосредственной близости от микрорезонатора для снижения паразитной емкости ВИЛ. Резонансная длина волны микрорезонатора смещена в коротковолновую сторону относительно пика усиления активной области на 10 нм (так называемая отрицательная отстройка спектра усиления относительно резонансной длины волны) с целью обеспечения высокого быстродействия при комнатной температуре.

Планарная технология изготовления ВИЛ с классической геометрией микрорезонатора включает следующие основные операции: формирование омического контакта $\mathrm{Ti} / \mathrm{Pt} / \mathrm{Au}$ к контактному слою $p^{+}$-GaAs, травление мезы до контактного слоя $n^{+}$-GaAs, формирование оксидной токовой апертуры с помощью селективного окисления апертурного слоя через вскрытые участки на боковых стенках мезы, формирование омического контакта $\mathrm{AuGe} / \mathrm{Ni} / \mathrm{Au}$ к контактному слою $n^{+}-\mathrm{GaAs}$, типа, планаризация развитой поверхности бензоциклобутеном (bisbenzocyclobutene, ВСВ), формирование площадок pи $n$-контактов в высокочастотной топологии. Для достижения компромисса между высоким быстродействием лазеров в режиме прямой токовой модуляции, сохранением низких пороговых токов и высокой дифференциальной эффективностью коэффициент отражения верхнего зеркала был оптимизирован экспериментальным путем с помощью мелкого травления приповерхностного слоя РБО [11].

Актуальный размер токовой оксидной апертуры для каждого исследованного ВИЛ контролировался двумя способами: анализом картины ближнего поля в допороговом режиме работы лазера и анализом контраста показателей преломления на границе оксид-полупроводник при ИК освещении. Вследствие небольшой анизотропии процесса селективного окисления слоев $\mathrm{AlGaAs}$ с высоким содержанием Al, оксидная токовая апертура имеет близкую к квадрату форму [12]. Было установлено, что область спонтанной электролюминесценции при малом уровне тока накачки хорошо вписывается в пределы области, определенной по границам контраста показателей преломления оксид-полупроводник. По-видимому, это обусловлено достаточно однородной инжекцией носителей в активную область вследствие наличия дополнительных потенциальных барьеров на гетерограницах относительно слабо легированных слоев РБО вблизи микрорезонатора. Исследования динамических характеристик ВИЛ проводились с помощью СВЧ зонда 
непосредственно на пластине, а для поляризационных исследований и измерения ширины линии излучения индивидуальные лазеры монтировались в тестовые корпуса (для уменьшения влияния механических напряжений от контактов зондов).

\section{3. Результаты и обсуждение}

Применение нескольких оксидных апертурных слоев для снижения емкости ВИЛ приводит к усилению волноводного эффекта в поперечном направлении (из-за увеличения контраста эффективного показателя преломления на внешних границах активной области), в результате чего в лазерах с характерным размером оксидной токовой апертуры более 3 мкм возникает многомодовая лазерная генерация через поперечные моды Лагерра-Гаусса.

На рис. 1 приведены ватт-амперные и спектральные характеристики ВИЛ с характерным размером токовой апертуры $\sim 2$ мкм, измеренные в непрерывном режиме работы при комнатной температуре. В приборах реализуется лазерная генерация вблизи 860 нм с пороговым током $\sim 0.56$ мА, дифференциальной эффективностью $\sim 0.65$ Вт/А и максимальной выходной оптической мощностью $\sim 1.75$ мВт при токе 5 мА. Согласно результатам анализа эволюции спектров генерации с током и температурой, тепловое сопротивление лазеров достигает $7 \mathrm{~K} / \mathrm{MB}$ вследствие достаточно низкой теплопроводности нижнего РБО $(\sim 25 \mathrm{BT} / \mathrm{M} \cdot K)$. С ростом тока накачки, обусловленные саморазогревом лазера тепловые эффекты (термическая линза, падение усиления и т.д.), ведут к снижению эффективности селекции фундаментальной моды. Тем не менее, одномодовый режим генерации с фактором подавления боковых мод (SMSR) > 30 дБ сохраняется во всем дипазоне токов накачки. Несмотря на вырождение фундаментальной моды резонатора по поляризации, поляризационные исследования выявили преобладание выделенного направления поляризации излучения ВИЛ с фактором подавления ортогональной поляризации (OPSR) > 15 дБ во всем рабочем диапазоне токов без каких-либо переключений поляризации, что, по-видимому, обусловлено электрооптическим эффектом или механическими напряжениями в комбинации с сложной формой оксидной токовой апертуры $[13,14]$.

Согласно соотношению Шавлова-Таунса [15] и с учетом особенностей поведения показателя преломления в полупроводниковых инжекционных лазерах [16], ширина линии излучения для одномодового ВИЛ может быть записана следующим образом:

$$
\Delta v_{L}^{S T}=\frac{\Gamma R_{\mathrm{sp}}^{\prime}}{4 \pi N_{p}}\left(1+\alpha^{2}\right),
$$

где $N_{p}$ - концентрация фотонов в рассматриваемой моде (т.е. общее количество фотонов $n_{p}$ в рассматриваемой моде, отнесенное к ее объему $V_{p}$, который в нашем случае полагается совпадающим с объемом

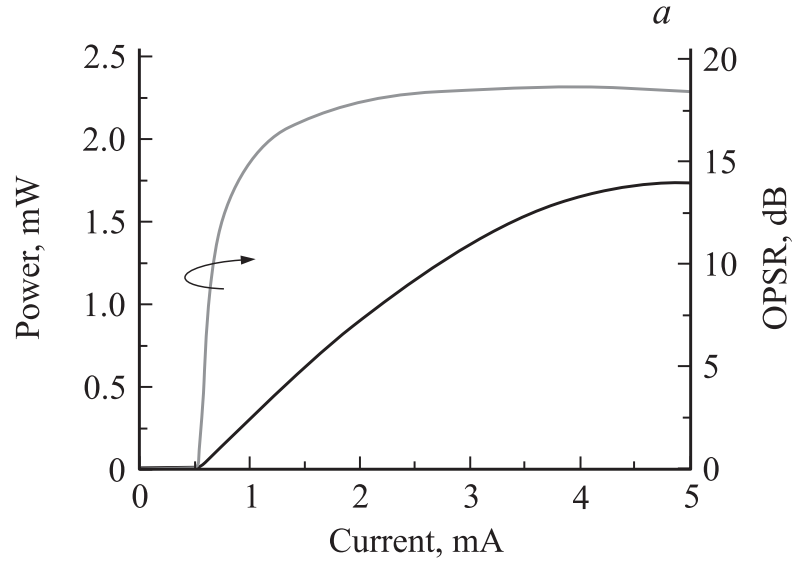

$b$

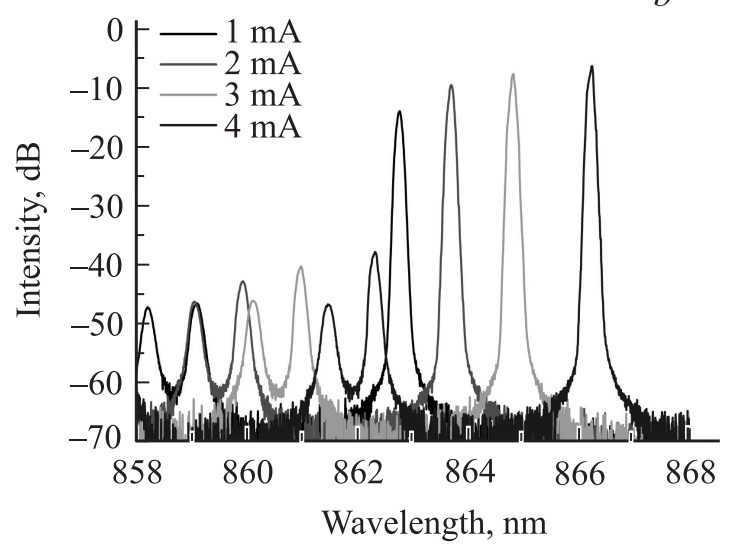

Рис. 1. ВИЛ с характерным размером токовой апертуры 2 мкм: $a-$ ватт-амперная характеристика и зависимость фактора подавления ортогональной поляризации OPSR от тока накачки; $b$ - спектры лазерной генерации при различных токах накачки. Измерения проведены при комнатной температуре.

микрорезонатора); $R_{\mathrm{sp}}^{\prime}-$ скорость спонтанного излучения, приходящегося на данную моду (т.е. количество фотонов, спонтанно излучаемых в рассматриваемую моду за единицу времени, отнесенное к объему активной области $\left.V_{a}\right), \Gamma-$ трехмерный фактор оптического ограничения (далее Г-фактор), $\alpha$ - фактор уширения спектральной линии (далее $\alpha$-фактор). Концентрация фотонов $N_{p}$ и скорость спонтанного излучения $R_{\mathrm{sp}}^{\prime}$ на пороге генерации могут быть выражены следующим образом [17]:

$$
\begin{gathered}
N_{p}=P / h v V_{p} v_{g} g_{\mathrm{th}}^{*} \eta_{0}, \\
R_{\mathrm{sp}}^{\prime}=v_{g} g_{\mathrm{th}}^{*} n_{\mathrm{sp}} / V_{a},
\end{gathered}
$$

где $P$ - выходная оптическая мощность, $h v-$ энергия фотонов, излучаемых на резонансной длине волны рассматриваемой моды, $v_{g}$ - групповая скорость $\left(\sim 8.5 \cdot 10^{9} \mathrm{~cm} / \mathrm{c}\right), g_{\mathrm{th}}^{*}-$ модальное пороговое усиление, $\eta_{0}$ - внешняя квантовая эффективность, $n_{\mathrm{sp}}-$ фактор инверсной заселенности (типично $\sim 1.5$ ). Поскольку Г-фактор выражается через объемы активной области 


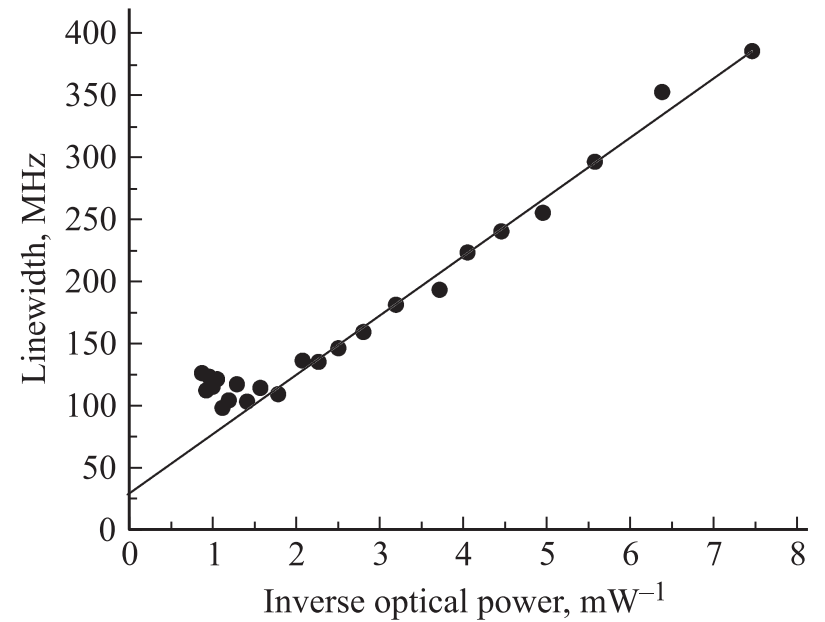

Рис. 2. Зависимость ширины линии излучения ВИЛ с характерным размером токовой апертуры $\sim 2$ мкм от обратной величины выходной оптической мощности. Измерения проведены при комнатной температуре.

и моды как $\Gamma=V_{a} / V_{p}$, выражение (1) может быть записано в следующем виде:

$$
\Delta v_{L}^{S T}=\frac{h v\left(v_{g} g_{\mathrm{th}}^{*}\right)^{2} n_{\mathrm{sp}} \eta_{0}}{4 \pi P}\left(1+\alpha^{2}\right) .
$$

На рис. 2 представлены результаты измерения ширины линии излучения $\Delta v_{L}$ ВИЛ с характерным размером токовой апертуры около 2 мкм, полученные с помощью сканирующего интерферометра Фабри-Перо Thorlabs SA-200 (разрешающая способность 7.5 МГц). Для подавления шумов, обусловленных нестабильностью источника питания и паразитной оптической обратной связью, использовался химический источник питания, а между лазером и интерферометром был введен оптический изолятор. На экспериментальной зависимости ширины линии генерации ВИЛ от уровня обратной выходной оптической мощности можно выделить два участка. На первом участке ширина линии излучения падает с ростом оптической мощности, однако при дальнейшем росте оптической мощности на втором участке наблюдается сильное отклонение $\Delta v_{L}$ от линейной зависимости, что может также приводить к уширению линии генерации одномодовых ВИЛ при прямой токовой модуляции [18]. В результате ширина линии излучения для исследуемых ВИЛ достигает минимального значения $\sim 110$ МГц при выходной оптической мощности $0.8 \mathrm{MBT}$ (рабочий ток $1.8 \mathrm{MA}$ ), что является типичной величиной для ВИЛ с классической геометрией микрорезонатора (без существенного увеличения его эффективной длины) $[8,19]$.

Зависимость ширины линии излучения ВИЛ на первом участке обратно пропорциональна величине выходной оптической мощности, что коррелирует с выражением (4), однако ее экстраполяция при стремлении значения оптической мощности к бесконечности дает ненулевое значение ширины линии излучения $\Delta v_{0} \approx 40$ МГц (так называемая остаточная ширина линии излучения). Данный факт, по-видимому, может быть обусловлен конкуренцией поперечных мод или связан с фликкер-шумом из-за флуктуации подвижностей носителей заряда [20]. В результате выражение для ширины линии излучения ВИЛ, адекватно описывающее экспериментальные зависимости, имеет вид

$$
\Delta v_{L}=\Delta v_{L}^{S T}+\Delta v_{0}
$$

Для оценки величины $\alpha$-фактора необходимо вычислить значения параметров $g_{\text {th }}^{*}$ и $\eta_{0}$. Модальное пороговое усиление компенсирует сумму внутренних оптических потерь и общих потерь на вывод излучения через оба зеркала микрорезонатора $T_{m}=-\ln \left(R_{F} R_{B}\right) / 2 L_{\mathrm{eff}}$, где эффективная длина $L_{\mathrm{eff}}(\sim 1.5$ мкм) учитывает конечную глубину проникновения электромагнитного поля в РБО. Поскольку отражательная способность нижнего (глухого) РБО в эксперименте всегда меньше 100\%, необходимо ввести поправочный коэффициент $F$, отражающий долю оптической мощности, выходящей через верхнее (выводное) РБО:

$$
F=\left(\frac{\sqrt{R_{B}}}{\sqrt{R_{F}}+\sqrt{R_{B}}}\right)\left(\frac{1-R_{F}}{1-\sqrt{R_{F} R_{B}}}\right) .
$$

В результате внешняя квантовая эффективность может быть записана как

$$
\eta_{0}=\frac{\eta_{d}^{F}}{F \eta_{j}} \frac{e}{h v}
$$

где $\eta_{d}^{F}$ - дифференциальная эффективность вывода излучения через верхнее РБО, $\eta_{i}$ - внутренняя квантовая эффективность (так называемая эффективность токовой инжекции), $e$ - заряд электрона. Расчеты коэффициентов отражения верхнего и нижнего РБО в рамках метода матриц переноса дают значения $R_{F} \approx 98.9 \%$ и $R_{B} \approx 99.99 \%$, что позволяет оценить значения $F \approx 0.99$ и $T_{m} \approx 37 \mathrm{~cm}^{-1}$. Внутренние оптические потери и внутренняя квантовая эффективность могут быть найдены в рамках подхода, описанного в работе [21]. В нашем случае для исследуемых одномодовых ВИЛ наблюдается увеличение внутренних оптических потерь $A_{\text {int }}$ до $21 \mathrm{~cm}^{-1}$ и падение эффективности токовой инжекции до $\sim 70 \%$ по сравнению с широкоапертурными приборами из-за диффузного рассеяния света на оксидных слоях и роста утечки носителей заряда при их повышенной концентрации. Подставляя (7) в (4) и учитывая соотношение (5), получаем для $\alpha$-фактора значение $\sim 3.4$, что коррелирует с аналогичными оценками $\alpha$-фактора для ВИЛ с активной областью на основе КЯ GaAs/AlGaAs [19] и напряженных КЯ $\mathrm{In}_{0.2} \mathrm{Ga}_{0.8} \mathrm{As} / \mathrm{GaAs}$ [22].

Аномальное уширение линии излучения с ростом мощностью ранее наблюдалось для одномодовых ВИЛ спектрального диапазона 1.55 мкм с туннельным переходом [23] и было объяснено нелинейностью усиления 


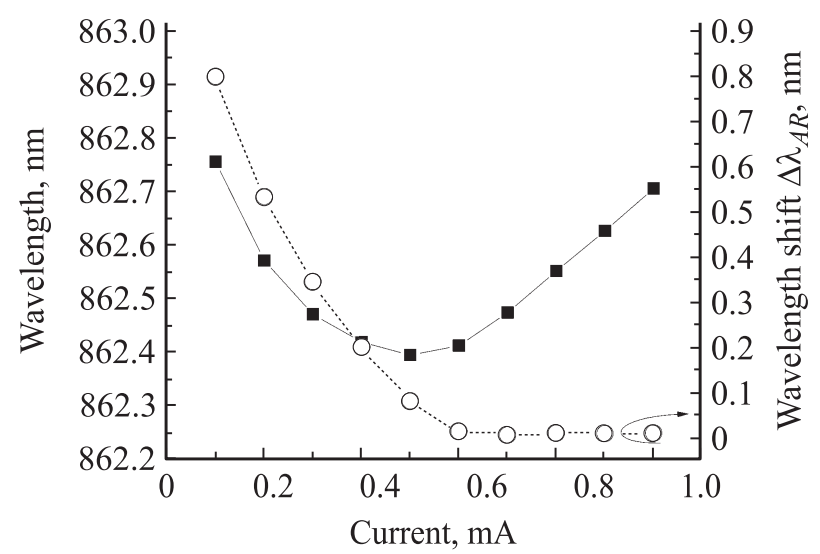

Рис. 3. Зависимость резонансной длины волны и сдвига резонансной длины волны $\Delta \lambda_{A R}$, обусловленного изменением показателя преломления активной области, от тока накачки для ВИЛ с характерным размером токовой апертуры $\sim 2$ мкм. Измерения проведены при комнатной температуре.

при наличии конкурирующих мод [24]. Однако в нашем случае сильный волноводный эффект на оксидной токовой апертуре ведет к фиксации модового состава лазера и формированию единого резервуара носителей для поперечных мод. Обнаруженное аномальное поведение ширины линии излучения на втором участке зависимости $\Delta v_{L}(P)$, по-видимому, связано с ростом $\alpha$-фактора при увеличении выходной мощности в условиях высокой плотности носителей и фотонов в микрорезонаторе [25]. В самом деле, $\alpha$-фактор равен отношению изменений вещественной и мнимой частей комплексного показателя преломления активной области лазера и может быть записан как $[16,17]$

$$
\alpha=\frac{4 \pi}{\lambda} \frac{\partial n / \partial N}{\partial g / \partial N},
$$

где $\lambda$ - длина волны излучения лазера, $\partial n / \partial N-$ вариация действительной части показателя преломления активной области с концентрацией носителей, $\partial g / \partial N-$ вариация усиления активной области с концентрацией носителей (дифференциальное усиление).

Согласно [26], изменение показателя преломления активной области, обусловленное изменением концентрации носителей в непрерывном режиме накачки, может быть найдено из анализа поведения резонансной длины волны ВИЛ в засисимости от тока (см. рис. 3). Наблюдаемый сдвиг резонансной длины волны $\Delta \lambda$ представляет собой суперпозицию коротковолнового сдвига резонансной длины волны $\Delta \lambda_{A R}$, обусловленного изменением показателя преломления активной области, и длинноволнового сдвига резонансной длины волны $\Delta \lambda_{S H}$, обусловленного саморазогревом лазера. Поскольку в первом приближении концентрация носителей фиксируется на пороге генерации, актуальный сдвиг $\Delta \lambda_{S H}$ может быть найден из анализа сдвига резонансной длины волны с рассеиваемой мощностью $P_{\text {dis }}$ выше порога генерации (в нашем случае $\partial \lambda / \partial P_{\text {dis }} \approx 0.42 \mathrm{HM} / \mathrm{MBT}$ ). На рис. 3 также представлена зависимость сдвига $\Delta \lambda_{A R}$ от тока $I$ для исследуемого ВИЛ, которая, согласно [27], может быть аппроксимирована выражением

$$
\Delta \lambda_{A R}=-k\left[\sqrt{I / I_{\mathrm{th}}}-1\right],
$$

где $k=1.35$ нм - итоговый сдвиг резонансной длины волны при достижении порогового тока $I_{\mathrm{th}}$, обусловленный изменением показателя преломления активной области. Тогда вариация показателя преломления активной области с концентрацией носителей может быть найдена из выражения [26]

$$
\frac{\partial n}{\partial N}=\frac{k n_{\mathrm{eff}} L_{\mathrm{eff}}}{\lambda d_{a} N_{\mathrm{th}}},
$$

где $n_{\mathrm{eff}}-$ эффективный показатель преломления $(\sim 3.5), d_{a}$ - общая толщина активной области, $N_{\text {th }}-$ пороговая концентрация носителей в активной области, которая может быть рассчитана с использованием выражения [17]

$$
N_{\mathrm{th}}=\frac{\eta_{i} I_{\mathrm{th}} \tau_{\mathrm{sp}}}{e V_{a}},
$$

где $\tau_{\mathrm{sp}}-$ время жизни носителей до порога. К сожалению, опубликованные данные по времени жизни носителей в напряженных КЯ InGaAs достаточно сильно разнятся. Так, согласно работе [28], значение $\tau_{\mathrm{sp}}$ для InGaAs КЯ слабо зависит от содержания In в пределах $5-15 \%$ и составляет $\sim 0.5$ нс. Однако в работе [29] было выявлено существенное влияние высоты потенциальных барьеров на значение $\tau_{\text {sp }}$ для КЯ $\mathrm{In}_{0.2} \mathrm{Ga}_{0.8} \mathrm{As} / \mathrm{AlGaAs}$, приводящее к его увеличению от $\sim 0.9$ до $\sim 4.2$ нс при уменьшении содержания $\mathrm{Al}$ от $20 \%$ до 0\%. Для получения достоверных данных была изготовлена тестовая гетероструктура с активной областью, аналогичной активной области исследуемого ВИЛ, для которой проведены измерения времени жизни носителей методом спектроскопии фотолюминесценции с временны́м разрешением. Анализ времени спада фотолюминесценции позволил оценить время жизни носителей в КЯ $\operatorname{In}_{0.08} \mathrm{Ga}_{0.92} \mathrm{As} / \mathrm{AlGaAs}$ на уровне $\sim 1.3 \mathrm{нс}$. Согласно выражению (11), пороговая концентрация носителей в активной области исследуемого ВИЛ достигает уровня $\sim 3.3 \cdot 10^{19} \mathrm{~cm}^{-3}$. Расчет вариации показателя преломления активной области с концентрацией носителей согласно выражению (10) дает значение $\sim 5.6 \cdot 10^{-21} \mathrm{~cm}^{3}$, что заметно ниже опубликованных данных для напряженных КЯ $\mathrm{In}_{0.2} \mathrm{Ga}_{0.8} \mathrm{As} / \mathrm{GaAs}$, но хорошо коррелирует с данными для КЯ $\mathrm{GaAs} / \mathrm{AlGaAs}$ [28].

Дифференциальное усиление активной области исследуемого ВИЛ может быть найдено из результатов малосигнального частотного анализа. На рис. 4 приведена зависимость резонансной частоты (или частоты релаксационных колебаний) от тока накачки, полученная с помощью аппроксимации экспериментальных амплитудночастотных характеристик в рамках стандартной модели 


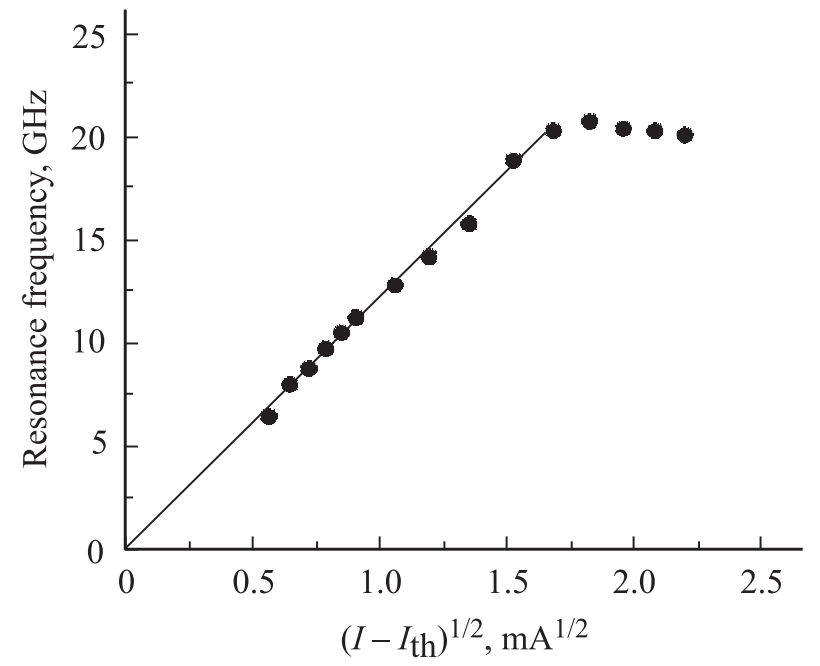

Рис. 4. Зависимость резонансной частоты от тока накачки $\sqrt{I-I_{\text {th }}}$ для ВИЛ с характерным размером токовой апертуры $\sim 2$ мкм. Измерения проведены при комнатной температуре.

частотного отклика инжекционного лазера. Скорость нарастания резонансной частоты с током (так называемый D-фактор) равна $\sim 12$ ГГц/мA $\mathrm{MA}^{1 / 2}$ и согласно [19] может быть записана как

$$
D=\frac{1}{2 \pi} \sqrt{\frac{\eta_{i} v_{g}}{e V_{p}}(\partial g / \partial N)} .
$$

Используя подход по оценке объема моды [31], дифференциальное усиление активной области можно оценить как $\sim 2.7 \cdot 10^{-16} \mathrm{~cm}^{2}$, что примерно в 3 раза меньше по сравнению с аналогичными оценками для широкоапертурных лазеров [11]. Расчет $\alpha$-фактора согласно выражению (8) дает значение $\sim 3$, что хорошо коррелирует с оценками $\alpha$-фактора из ширины линии излучения лазера. Однако дифференциальное усиление зависит как от концентрации носителей, так и от спектрального рассогласования спектра усиления и резонансной длины волны [22]. С одной стороны, рост концентрации носителей ведет к падению дифференциального усиления и, как следствие, к росту $\alpha$-фактора. С другой стороны, сдвиг резонансной длины волны в коротковолновую сторону спектра усиления (относительно пика) при фиксированной концентрации носителей ведет к росту дифференциального усиления и, как следствие, к уменьшению $\alpha$-фактора. Падение усиления требует увеличения концентрации носителей для достижения порога генерации, тем самым частично компенсируя падение $\alpha$-фактора. Таким образом, малый объем микрорезонатора (малый размер токовой апертуры) в условиях высоких внутренних потерь ведет к высокой концентрации носителей в микрорезонаторе, что, в свою очередь, быстро переводит лазер в режим насыщения усиления с ростом тока накачки и росту $\alpha$-фактора, приводящему к уширению линии излучения.

\section{4. Заключение}

В работе проведены детальные исследования одномодовых ВИЛ спектрального диапазона 850 нм на основе КЯ InGaAs/AlGaAs с характерным размером токовой апертуры $\sim 2$ мкм. В лазерах получена одномодовая лазерная генерация с подавлением ортогонально-поляризованной моды > 15 дБ. Ширина линии излучения лазера сначала уменьшается обратно пропорционально с ростом выходной оптической мощности и достигает своего минимума $\sim 110$ МГц при 0.8 мВт. Однако в условиях повышенной концентрации носителей в микрорезонаторе (из-за малого размера и высоких внутренних оптических потерь) лазер переходит в режим насыщения усиления, что ведет к падению дифференциального усиления с ростом тока накачки, росту $\alpha$-фактора и увеличению ширины линии излучения.

Была проведена оценка величины $\alpha$-фактора двумя различными способами. Оценка $\alpha$-фактора из экспериментальной зависимости ширины линии излучения от оптической мощности с учетом остаточной ширины линии излучения $\Delta v_{0} \approx 40$ МГц дает значение $\sim 3.4$. Оценка $\alpha$-фактора исходя из значений вариации показателя преломления активной области с концентрацией носителей и дифференциального усиления активной области дает значение $\sim 3$. Близкие значения $\alpha$-фактора, полученные двумя независимыми методами, позволяют сделать заключение об адекватности произведенных оценок. Обнаруженные эффекты и полученные данные могут быть использованы для последующей оптимизации конструкции одномодовых ВИЛ с целью минимизации ширины линии лазерного излучения.

Исследованные лазеры являются результатом совместного проекта между VI- Systems GmbH и ООО Коннектор Оптикс.

\section{Список литературы}

[1] R. Michalzik. VCSELs: Fundamentals, Technology and Applications of Vertical-Cavity Surface-Emitting Lasers (Berlin, Springer-Verlag, 2013).

[2] P. Moser, P. Wolf, G. Larisch, H. Li, J.A. Lott, D. Bimberg. Proc. SPIE, 9001, 900103 (2014).

[3] E. Haglund, P. Westbergh, J.S. Gustavsson, E.P. Haglund, A. Larsson, M. Geen, A. Joel. Electron. Lett., 51 (14), 1096 (2015).

[4] P.D.D. Schwindt, B. Lindseth, S. Knappe, V. Shah. J. Kitching, L.-A. Liew. Appl. Phys. Lett., 90, 081102 (2007).

[5] M. Prouty, A. Miniature. Wide Band Atomic Magnetometer, SERDP Project MR-1568, Geometrics (2011).

[6] A. Pruijmboom, M. Schemmann, J. Hellmig, J. Schutte, H. Moench, J. Pankert. Proc. SPIE, 6908, 69080I (2008).

[7] L. Knappe, V. Shah, P.D.D. Schwindt, L. Hollberg, J. Kitching, L.A. Liew, J. Moreland. Appl. Phys. Lett., 85, 1460 (2004).

[8] D.K. Serkland, K.M. Geib, G.M. Peake, R. Lutwak, A. Rashed, M. Varghese, G. Tepolt, M. Prouty. Proc. SPIE, 6484, 648406 (2007). 
[9] D.K. Serkland, G.A. Keeler, K.M. Geib, G.M. Peake. Proc. SPIE, 7229, 722907 (2009).

[10] S.B. Healy, E.P. O’Reilly, J.S. Gustavsson, P. Westbergh, E. Haglund, A. Larsson, A. Joel. IEEE J. Quant. Electron., 46, 506 (2010).

[11] M.A. Bobrov, S.A. Blokhin, N.A. Maleev, A.G. Kuzmenkov, A.A. Blokhin, Yu.M. Zadiranov, S.I. Troshkov, N.N. Ledentsov, V.M. Ustinov. J. Phys.: Conf. Ser., 643, 012044 (2015).

[12] K.D. Choquette, K.M. Geib, C.I.H. Ashby, R.D. Twesten, O. Blum, H.Q. Hou, D.M. Follstaedt, B.E. Hammons, D. Mathes, R. Hull. IEEE J. Select. Topics in Quant. Electron., 3, 916 (1997).

[13] M.P. van Exter, A.K. Jansen van Doorn, J.P. Woerdman. Phys. Rev. A, 56, 845 (1997).

[14] F. Monti diSopra, M. Brunner, R. Hövel. Photon. Technol. Lett., 14, 1034 (2002).

[15] A.L. Schawlow, C.H. Townes. Phys. Rev., 112, 1940 (1958).

[16] C.H. Henry. IEEE J. Quant. Electron., 18, 259 (1982).

[17] K. Petermann. Laser diode modulation and noise (Kluwer Academic, 1991).

[18] N.N. Ledentsov, J.A. Lott, J.-R. Kropp, V.A. Shchukin, D. Bimberg, P. Moser, G. Fiol, A.S. Payusov, D. Molin, G. Kuyt, A. Amezcua, L.Y. Karachinskiy, S.A. Blokhin, I.I. Novikov, N.A. Maleev, C. Caspar, R. Freund. Proc. SPIE, 8276, 82760K (2012).

[19] D. Kuksenkov, S. Feld, C. Wilmsen, H. Temkin, S. Swirhun, R. Leibenguth. Appl. Phys. Lett., 66, 277 (1995).

[20] L.A. Coldren, S.W. Corzine, M.L. Mašanović. Diode Lasers and Photonic Integrated Circuits (N.Y., Wiley, 2012).

[21] G.M. Yang, M.H. Mac Dugal, V. Pudikov, P.D. Dapkus. Photon. Technol. Lett., 7, 1228 (1995).

[22] D. Summers, P. Dowd, I.H. White, M.R.T. Tan. Photon. Technol. Lett., 7, 736 (1995).

[23] A. Bacou, A. Rissons, J.-C. Mollier. Proc. SPIE, 6908, 69080F (2008).

[24] KU. Krüger, K. Petermann. IEEE J. Quant. Electron., 24, 2355 (1988).

[25] H. Halbritter, R. Shau, F. Riemenschneider, B. Kögel, M. Ortsiefer, J. Rosskopf, G. Böhm, M. Maute, M.-C. Amann, P. Meissner. Electron. Lett., 40, 1266 (2004).

[26] K. Kishino, S. Aoki, Y. Suematsu. J. Quant. Electron., QE-18, 343 (1982).

[27] K. Stubkjaer, Y. Suematsu, M. Asada, S. Arai, A.R. Adams. Electron. Lett., 16, 895 (1980).

[28] M.H. Moloney, J. Hegarty, L. Buydens, P. Demeester, R. Grey, J. Woodhead. Appl. Phys. Lett., 62, 3327 (1993).

[29] A.P. Ongstad, D.J. Gallant, G.C. Dente. Appl. Phys. Lett., 66, 2730 (1995).

[30] M. Usami, H. Sakata, Y. Matsushima. Proc. $19^{\text {th }}$ Intern. Symp. Gallium Arsenide and Related Compounds (1992) p. 803.

[31] C. H. Wu, F. Tan, M. Feng, N. Holonyak,jr. Appl. Phys. Lett., 97, 091103 (2010)

\section{Linewidth and $\alpha$-factor in $850-\mathrm{nm}$ single-mode vertical-cavity surface-emitting laser based on quantum wells InGaAs/AIGaAs}

S.A. Blokhin ${ }^{1}$, M.A. Bobrov' ${ }^{1}$, A.A. Blokhin ${ }^{1}$, A.G. Kuzmenkov', A.P.Vasil'ev 2,1, Y.M. Zadiranov', E.A. Evropeytsev ${ }^{1}$, A.V. Sakharov ${ }^{1}$, N.N. Ledentsov ${ }^{3}$, L.Ya. Karachinsky, ${ }^{4,1}$, A.M. Ospennikov ${ }^{5}$, N.A. Maleev ${ }^{1}$, V.M. Ustinov ${ }^{2,1,6}$

${ }^{1}$ loffe Institute, 194021 St. Petersburg, Russia

${ }^{2}$ Submicron Heterostructures for Microelectronics,

Research \& Engineering Center of the Russian Academy of Sciences, 194021 St. Petersburg, Russia

${ }^{3}$ VI Systems GmbH, Berlin, Germany, D-10623

${ }^{4}$ Connector Optics LLC, 194292 St. Petersburg, Russia

${ }^{5}$ Russian Institute of Radionavigation and Time RIRT, 192012 St. Petersburg, Russia

${ }^{6}$ Peter the Great St. Petersburg Polytechnic University, 195251 St. Petersburg, Russia

Abstract Studies of linewidth in $850-\mathrm{nm}$ singlequantum wellsmode vertical-cavity surface-emitting lasers based on InGaAs/AlGaAs quantum wells are presented. Lasers with $2 \mu \mathrm{m}$ oxide current aperture size exhibit minimum linewidth of $110 \mathrm{MHz}$ at output power of $0.8 \mathrm{~mW}$. With a further increase in the output power, an anomalous emission linewidth broadening is observed, which is apparently caused by an increase in $\alpha$-factor due to a decrease in the differential gain of the active region under conditions of an increased carrier concentration and high internal optical losses in the cavity. The $\alpha$-factor is estimated by two independent methods.

Редактор Л.В. Шаронова 